\title{
Cardiac Postconditioning
}

\author{
Pasquale Pagliaro and Claudia Penna
}

\begin{abstract}
In the heart, ischemia/reperfusion damage occurs mainly during the first minutes of reperfusion. Recently, it has been shown that the heart can be protected against the extension of ischemia/reperfusion injury if brief coronary occlusions are performed just at the beginning of the reperfusion. This procedure has been called postconditioning (PostC). It can also be elicited by pharmacological intervention, that is, pharmacological PostC. In particular, PostC limits infarct size, apoptosis, endothelial dysfunction, neutrophil adherence, and arrhythmias. Similar to preconditioning, PostC may trigger signaling pathways, including reperfusion injury salvage kinase and survivor activating factor enhancement pathways. PostC-induced protection also involves intracellular acidosis and early redox-sensitive mechanisms. However, controversies exist on the nature of receptors and main pathway(s) involved in PostC. Protective pathways activated by PostC appear to converge on mitochondria and, in particular, on mitochondrial permeability transition pores. Preliminary clinical data indicate that drugs targeting mitochondrial permeability transition pore or reperfusion injury salvage kinases may confer benefits to patients with acute myocardial infarction above that provided by myocardial reperfusion alone. Future studies must define the principal protective cascades, the interdependence of the signaling pathways, and the optimal pharmacological target and agent(s) for protection. These studies must also consider the possible confounding effects of comorbidities and their drug treatments. Antioxid. Redox Signal. 14, 777-779.
\end{abstract}

\section{Introduction}

$\mathbf{M}$ YOCARDIAL INFARCTION is the major cause of mortality in industrialized countries. Therefore, protecting the heart from ischemia- and reperfusion-induced injury represents the greatest challenge of modern cardiology.

It is now clear that the mammalian heart can partially or totally recover its pump function only if coronary flow is promptly restored in the ischemic area. In fact, reperfusion is the crucial treatment to reduce all manifestations of postischemic injury. On the contrary, when it is not possible to start an early reperfusion, the progression of ischemic-induced alterations causes irreversible damage that leads inexorably to contractile dysfunction and cell death. Although early reperfusion is the only way to salvage an ischemic cell, during the crucial early moments of reperfusion, significant irreversible cell damage is initiated, which is referred to as reperfusion injury. These include cell death by necrosis and apoptosis, contractile dysfunction, arrhythmias, microvascular injury or "no-reflow," and inflammatory responses $(7,11)$. Therefore, both ischemia and reperfusion contribute to organ injury. However, different animal species can tolerate extremely different levels of oxygenation. Cardioprotective interventions may render the mammalian heart more resistant to ischemia/reperfusion challenges. An important issue of cardioprotection research is to understand the mechanisms underlying heart vulnerability and resistance against ischemia. To address this issue, the present forum presents an elegant review on the tolerance of vertebrate hearts to hypoxia and anoxia in an evolutionary and comparative perspective (10). In this respect, it is particularly intriguing that cardioprotective interventions against reperfusion injury can be performed either before infracting/index ischemia (preconditioning) or in the early moments of reperfusion, which follows ischemia [postconditioning (PostC)].

\section{PostC Cardioprotection}

Cardioprotective interventions can be pharmacological or mechanical (brief periods of ischemia/reperfusion or gentle/gradual reperfusion) in nature. In fact, paradoxically, survival mechanisms in the heart can be triggered by short, nonlethal periods of ischemia and reperfusion, applied either before (ischemic preconditioning) or immediately after (ischemic or mechanical PostC) an infarcting/index ischemia. The present forum analyzes the accumulating amount of evidence that lethal reperfusion injury may be reduced by ischemic or pharmacological PostC in animals (1-11) and in

Department of Clinical and Biological Sciences, University of Turin, Orbassano, Italy. 
small-sized clinical trials (6). Vinten-Johansen and collaborators discuss the protective effect of PostC against infarct size, arrhythmias, stunning, and vascular dysfunction (11). PostC activates signaling pathways, which involve triggers, mediators, and end-effector(s): the contribution of each of these players may vary depending on the experimental model (cell type, species, in vivo, ex vivo, and in vitro) and the PostC stimulus used (Fig. 1). The triggers, with particular attention paid to adenosine and its receptors, are considered in the review by Cohen and Downey (2). The exact nature of receptors involved in triggering protection remains a matter of controversy $(2,7)$. The mediators, mostly protein kinases, are clearly discussed by Hausenloy et al. (4). In this review (4), the reperfusion injury salvage kinase pathway and the more recently described survivor activating factor enhancement pathways, two apparently distinct signaling pathways, are considered. In these two reviews $(2,4)$, the authors bring upto-date useful concepts on mechanisms in a constantly changing field. Controversy on the role of reperfusion injury salvage kinase is also discussed $(4,7)$. Inserte et al. (5) elegantly discuss interactions between intracellular acidosis and endogenous protection signaling. Low intracellular $\mathrm{pH}$, in fact, inhibits many mechanisms involved in reperfusion injury, including opening of mitochondrial permeability transition pore (mPTP) and activation of calpains. Accumulating evidences show the importance of acidotic environment in early

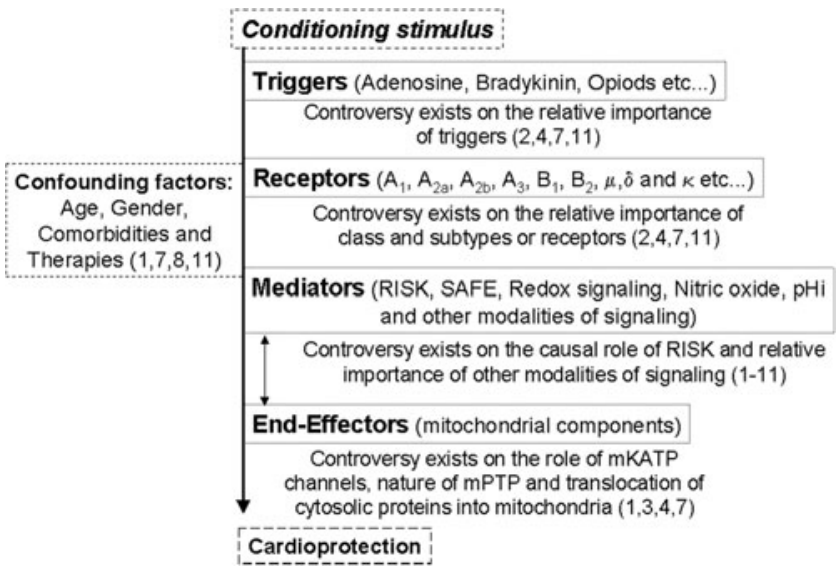

FIG. 1. Protective cascade and signaling pathways, which involve triggers and mediators converging on mitochondria, where end-effector(s) reside. Controversies and confounding factors are highlighted. Future studies must deal with these controversies and solve the conundrum of confounding factors in the setting of aging, diabetes, hypertension, and other diseases. The double-headed arrow indicates the cross-talk between mediators and end-effectors. In particular, reactive oxygen species generated by mitochondria may influence other cytosolic components (e.g., kinases, phosphatases, and other enzymes), which in turn may influence mitochondrial function. The numbers in parentheses are references that address in more detail the various points of the protective cascade and discuss the controversy present in the literature. Adenosine receptors subtypes: $A_{1}, A_{2 a}, A_{2 b}$, $\mathrm{A}_{3}$; bradykinin receptors subtypes: $\mathrm{B}_{1}, \mathrm{~B}_{2}$; opiods receptors subtypes: $\mu, \delta, \kappa . \mathrm{mKATP}$, mitochondrial $\mathrm{K}^{+}$ATP channels; $\mathrm{mPTP}$, mitochondrial permeability transition pore; $\mathrm{pHi}$, intracellular $\mathrm{pH}$; RISK, reperfusion injury salvage kinase; SAFE, survivor activating factor enhancement. reperfusion for cardioprotection. The protective molecular pathways are incompletely understood but it is very likely that they converge on mitochondria, where suppression of $\mathrm{mPTP}$ opening during early reperfusion is considered the key event preventing cell death at reperfusion (Fig. 1). The end effectors within mitochondria and, in particular, the regulation of MPTP and its implication for cell pathology are discussed by Di Lisa et al. (3). The review by Boengler et al. (1) clearly discusses how modifications of mitochondrial function and mPTP opening contribute to cardiac pathophysiology, specifically during reperfusion. The role of mitochondria in loss of PostC protection in diseased or aged myocardium is also discussed (1). The importance of mitochondria is outlined by all the authors of the forum, as many components of these organelles are involved in cardioprotective mechanisms. Different aspects of the role of these organelles are analyzed in more detail by Boengler et al. (1), Di Lisa et al. (3), and Pagliaro et al. (7). These insights have been already translated to the treatment of patients with reperfused acute myocardial infarction (6). Both Post $C$ with ischemia and with drugs have been used in humans, and useful clinical information is given in the review by Ovize and coworkers (6). Intriguingly, there appear to be redundant cardioprotective pathways; these pathways, their interactions, and their influences by redox environment are discussed by Pagliaro et al. (7). In particular, redox signaling is suggested to play a role in both preconditioning and PostC during the initial part of reperfusion that follows the infarcting ischemia (7).

The extent of protection by ischemic PostC is influenced by many variables including the duration of index ischemia, the PostC protocol, the species, gender, and even interindividual variations. The influence of confounding factors (Fig. 1), including the classical coronary artery disease risk factors, drug treatments, gender, and age, on PostC efficacy is discussed by Vinten-Johansen et al. (11). The original article by Przyklenk et al. (8) shows how diabetic conditions and therapies may influence PostC efficacy. Some features of pharmacological PostC are also considered in the review of Losano and coworkers (9), where the unique aspects of the protective effects of Apelin are analyzed.

\section{Conclusions and Open Questions}

Methods to protect the heart during reperfusion are of great clinical interest. It is now clear that protective interventions must be applied early on, and the first seconds to minutes have the most significant impact on "acute" reperfusion injury, as shown in many experimental studies reported in the reviews of the present forum. There is indeed the necessity to define the optimal protective protocol for a given duration of index ischemia and for disparate clinical manifestations. It is also of utmost importance to define the principal protective cascades, the interdependence of the signaling pathways, and the optimal pharmacological target and agent (classical or innovative) for protection. This should be done in preclinical and clinical studies, which must also consider the possible comorbidities and their drug treatments. In fact, understanding the modifications of the protective pathways in the presence of comorbidities and their therapies is also of paramount importance. Finally, clinical trials, including large multicenter studies, to determine the impact of ischemic and pharmacological PostC on patient outcomes are mandatory. 
These studies must include an appropriate number of patients to allow for accurate statistical stratification into subsets according to individual comorbidities, multiple comorbidities, and medications used to treat acute or chronic coronary artery disease.

\section{Acknowledgments}

The authors thank Regione Piemonte, INRC (Bologna, Italy), MIUR (Torino, Italy; PRIN 2006, 2008), and Compagnia di San Paolo (Torino, Italy) for financial support. The authors thank Prof. Donatella Gattullo for her invaluable support and Dr. Jennifer M. Lee for language revision.

\section{References}

1. Boengler K, Heusch G, and Schulz R. Mitochondria in postconditioning. Antioxid Redox Signal 14: 863-880, 2011.

2. Cohen MV and Downey JM. Ischemic postconditioning: from receptor to end-effector. Antioxid Redox Signal 14: 821831, 2011.

3. Di Lisa F, Canton M, Carpi A, Kaludercic N, Menabò R, Menazza S, and Semenzato M. Mitochondrial injury and protection in ischemic pre- and post-conditioning. Antioxid Redox Signal 14: 881-891, 2011.

4. Hausenloy DJ, Lecour S, and Yellon DM. Reperfusion Injury Salvage Kinase and Survivor Activating Factor Enhancement prosurvival signaling pathways in ischaemic postconditioning: two sides of the same coin. Antioxid Redox Signal 14: 893-907, 2011.

5. Inserte J, Ruiz-Meana M, Rodríguez-Sinovas A, Barba I, and Garcia-Dorado D. Contribution of delayed intracellular $\mathrm{pH}$ recovery to ischemic postconditioning protection. Antioxid Redox Signal 14: 923-939, 2011.

6. Ivanès F, Rioufol G, Piot C, and Ovize M. Postconditioning in acute myocardial infarction patients. Antioxid Redox Signal 14: 811-820, 2011.

7. Pagliaro P, Moro F, Tullio F, Perrelli M-G, and Penna C. Cardioprotective pathways during reperfusion: focus on redox signaling and other modalities of cell signaling. Antioxid Redox Signal 14: 833-850, 2011.
8. Przyklenk K, Maynard M, Greiner DL, and Whittaker P. Cardioprotection with postconditioning: loss of efficacy in murine models of type-2 and type-1 diabetes. Antioxid Redox Signal 14: 781-790, 2011.

9. Rastaldo R, Cappello S, Folino A, and Losano G. Effect of Apelin-Apelin receptor system in postischaemic myocardial protection: a pharmacological postconditioning tool? Antioxid Redox Signal 14: 909-922, 2011.

10. Tota B, Angelone T, Mancardi D, and Cerra MC. Hypoxia and anoxia tolerance of vertebrate hearts: an evolutionary perspective. Antioxid Redox Signal 14: 851-862, 2011.

11. Vinten-Johansen J, Granfeldt A, Mykytenko J, Undyala VV, Dong Y, and Przyklenk K. The multidimensional physiological responses to postconditioning. Antioxid Redox Signal 14: 791-810, 2011.

Address correspondence to: Dr. Pasquale Pagliaro Dipartimento di Scienze Cliniche e Biologiche Università di Torino Regione Gonzole 10 Orbassano (TO) 10043 Italy

E-mail: pasquale.pagliaro@unito.it

Date of first submission to ARS Central, July 28, 2010; date of acceptance, August 13, 2010.

$\begin{aligned} & \text { Abbreviations Used } \\ & \mathrm{mKATP}=\text { mitochondrial } \mathrm{K}^{+} \text {ATP channels } \\ & \mathrm{mPTP}=\text { mitochondrial permeability transition } \\ & \quad \text { pore } \\ & \mathrm{pHi}=\text { intracellular } \mathrm{pH} \\ & \mathrm{PostC}=\text { postconditioning } \\ & \mathrm{RISK}=\text { reperfusion injury salvage kinase } \\ & \mathrm{SAFE}=\text { survivor activating factor enhancement }\end{aligned}$


